\title{
Return to sport following hip injury
}

\author{
Pete Draovitch • Robert A. Maschi • Jessica Hettler
}

Published online: 29 February 2012

(C) Springer Science+Business Media, LLC 2012

\begin{abstract}
Returning to both recreational and competitive sport requires the patient demonstrate the ability to perform their activity without pain, without compensation and without apprehension. The primary focus of this article will be to provide progressive weight bearing phased treatment solutions and both objective and empirical return to play testing suggestions. In addition to satisfactorily completing the test battery with maximum effort, the patient must demonstrate the ability to meet the demands for competing within their respective sport specific environment. Returning to competition will most likely include early phase movement screening and clinical clearance followed by late phase athletic and field testing. Hip structure will and must influence training program design. It is therefore important to recognize that indications for return to play must not focus on a single rehabilitation or exercise variable, but rather a combination of clinical, functional and sport specific demands.
\end{abstract}

Keywords Femoroacetabular impingement $(\mathrm{FAI}) \cdot$ Clinical and field testing $\cdot$ Transitional progression $\cdot$ Sport specific training

P. Draovitch $(\square)$

Center for Hip Preservation, Hospital for Special Surgery Sports

Rehab and Performance Center,

New York, NY, USA

e-mail: draovitchp@hss.edu

\section{R. A. Maschi}

Department of Physical Therapy and Rehabilitation Science,

Drexel University,

Philadelphia, PA, USA

e-mail: robmaschi@drexel.edu

\section{J. Hettler}

Hospital for Special Surgery Sports Rehab

and Performance Center,

New York, NY, USA

e-mail: hettlerj@hss.edu

\section{Introduction}

Returning to the competitive arena provides challenges for both the athlete and all those responsible for overseeing the transition from the training room to the field of play. The initial discussions at the time of injury must include what will be physically required before returning to play and what will be the safest and most effective avenues to ensure a progressive return. The primary focus of this article will be to provide progressive weight bearing phased treatment solutions and both objective and empirical return to play testing suggestions. Evidenced-based outcomes have led to identifying specific objective clinical markers that dictate when a patient is cleared to return to their sport. This is in addition to satisfactorily completing field and sport specific tests. Many times during the process there may be a limited awareness of the patient's exercise tolerance or threshold. The patient may be able to participate without restriction for a period of time, but eventually lacks the physiological or force load acceptance capacity to continue without compensation. At this point in the rehabilitation process, the patient's competitive on the field time may be restricted, however other progressive weight bearing exercises will be implemented to continue to develop their stamina in a controlled loading environment.

\section{Rehabilitation transitional progressions}

Aquatic rehabilitation

During the rehabilitation process there are times when having the ability to unload a joint can assist with faster healing and return to play. It allows for introduction of normal biomechanics and fluid mobility in a joint. Buoyancy of water (chest deep equals $40 \%$ total body weight, waist deep equals $60 \%$ total body weight) is a counterforce to gravity on the body, 
which allows for training to be completed with higher repetitions resulting in increased physical benefits $[1,2]$. Recent literature does indicate that running speeds should be monitored since the level of immersion may not be indicative of the amount of force generated during running in both waist and chest high immersion levels. [3•] Utilization of aquatic training assists in injury prevention, safe training environment, and improved overall performance [1].

Aquatic rehabilitation assists in overall gains in range of motion (ROM), coordination of movement, and cardiorespiratory endurance. Different positions and equipment may be added to the water to assist in achievement of goals. Deep water running demonstrates similar results in cardiovascular fitness when compared to land based running (maximal oxygen uptake within $5 \%$ of land based scores) [4]. Electromyographic (EMG) studies have shown an increase in maximal voluntary contractions of the gluteus medius and adductor longus to during deep water running. This is thought to be due stabilization of the pelvis [5]. One should proceed with caution with deep water running if the patient already presents with increased tone of the adductor longus, which may be attempting to compensate for weak hip flexion or frontal plane stability insufficiency.

Decreasing water depth assists in normalizing the gait cycle by increasing axial loading throughout the lower extremity [6]. During anterior cruciate ligament reconstruction (ACLR) rehabilitation, patients demonstrate a reduction in swelling and greater functional movements in the aquatic environment. Education on closed chain exercises and progressive weight-bearing are achieved earlier in the aquatic environment. Also, open chain exercises initiated in the water will minimize the excessive loading on the joint and safely provide accommodating resistance throughout the motion. Most important, aquatic rehabilitation assists in improving joint mechanics and strengthening, allowing for safe transition to land-based exercises [7].

\section{Unloaded treadmills}

A weight unloading treadmill or Lower Body Positive Pressure (LBPP) treadmill such as the Alter $\mathrm{G}^{\circledR}$ (AlterG AntiGravity Treadmill, AlterG Incorporated, Fremont, CA) can be a useful tool in the transition from early postoperative phases up to the return to sport phase of rehabilitation. The Alter $G^{\circledR}$ and similar LBPP devices achieve un-weighting by using air in a pressure controlled chamber that lifts the user up from the treadmill, thereby decreasing ground reaction force (GRF) and load transmitted through tissues of the lower limbs. The air pressure inside the chamber can be increased above ambient pressure using an air compressor in the device. The pressure differential causes an axially directed buoyant force. The net ground reaction force then becomes the difference between the weight of the user and this axial force. Increasing axial force (un-weighting) will decrease GRF [8]. The user can be un-weighted from $99 \%$ of body weight down to $20 \%$ of body weight in $1 \%$ increments.

The benefits of unloading include allowing early initiation of weight bearing and closed kinetic chain activities in a safe environment for healing tissue. Increased vertical GRF and vertical loading have been associated with increased risk for stress injury or overuse injury [9-11]. Decreasing these loads will allow safe acceleration of functional activity. The patient, while partial weight bearing, may begin to normalize gait without the use of assistive devices and start to re-establish normal neuromuscular activation patterns. Weight can be added incrementally to allow gradual adaptation to occur without overloading healing tissue. Initial treatment may consist of walking at $1-2 \mathrm{mph}$ for $5 \mathrm{~min}$. This is a $50 \%$ reduction in average walking speed which is approximately $3.0 \mathrm{mph}$. Speed and weight can be gradually increased eventually progressing to running as healing advances and strength improves. Using LBPP has been shown to significantly decrease pain during ambulation in patients who recently underwent arthroscopic meniscectomy or ACLR [12] and has been utilized in rehabilitation program following microfracture of the knee [13].

LBPP has been investigated regarding its effects on loading and gait parameters during walking and running. Ground reaction forces are reduced for running and walking at all levels of un-weighting using the LBPP treadmill versus full body weight $[8,12,14-16]$. Running mechanics are not significantly affected with un-weighting with respect to stride length and knee and ankle sagittal plane ROM [8, 17, 18]. There is no significant change in muscle EMG firing patterns (bicep femoris, rectus femoris, tibialis anterior, and gastrocnemius) while running in the LBPP treadmill $[17,18]$. This will allow normal muscle firing patterns to be reinforced even before the patient is able to fully load the limb. Although firing patterns remain consistent, muscle EMG peak amplitude is decreased with un-weighting [17, 18]. In the un-weighted condition there is less demand on antigravity muscles of the leg. This condition allows for closed chain functional activity early with muscles that have been weakened by trauma or surgery. Muscle EMG peak amplitude can be increased (increasing muscle demand) by increasing speed of the LBPP treadmill $[17,18]$.

Tables 1 and 2 are samples of progressions for a patient utilizing a LBPP treadmill during rehabilitation following hip arthroscopy. Note: this is only an illustrative guide and must be adapted to individual patient condition and response to treatment.

It has been suggested to make the transition out of the LBPP treadmill for full weight bearing once the patient is able to tolerate $85 \%$ weight bearing $[19,20]$. At this point training may continue on a conventional treadmill or with 
Table 1 Post-operative progression using a LBPP treadmill (2-3x/ week)

\begin{tabular}{lccc}
\hline Post Op week & Time (min) & Speed (mph) & Body weight \\
\hline 1 & 5 & 1.5 & $40 \% \mathrm{BW}$ \\
2 & 5 & 2.0 & $40 \% \mathrm{BW}$ \\
3 & 5 & 2.0 & $50 \% \mathrm{BW}$ \\
4 & 5 & 3.0 & $50 \% \mathrm{BW}$ \\
5 & 10 & 3.5 & $50 \% \mathrm{BW}$ \\
6 & 10 & 3.5 & $55 \% \mathrm{BW}$ \\
7 & 10 & 3.5 & $60 \% \mathrm{BW}$ \\
8 & 10 & 3.5 & $65 \% \mathrm{BW}$ \\
9 & 8 & 4.0 & $70 \% \mathrm{BW}$ \\
10 & 8 & 4.0 & $75 \% \mathrm{BW}$ \\
11 & 8 & 4.0 & $80 \% \mathrm{BW}$ \\
12 & 8 & 4.0 & $85 \% \mathrm{BW}$ \\
\hline
\end{tabular}

over ground running. The LBPP treadmill may be useful in the return to sport phase as an adjunct to therapy. It can be used for conditioning and performance enhancement with the benefit of reducing risk associated with large volume training. It has been demonstrated that increasing velocity can cause significant increase in metabolic demand sufficient to improve cardiovascular fitness while gaining the benefit of decreased load and ground reaction force [16]. A portion of training may be done on the LBPP to supplement over ground training. It has also been suggested that "over speed" training or fast velocity running is beneficial for improving cardiovascular and neuromuscular function [21]. It has been demonstrated that this type of training is effective at producing greater rates of muscular force generation $[22,23]$. Traditional "over speed" training must be approached cautiously because of increased peak ground reaction forces that accompany running at fast velocity. These forces can be mitigated with use of the LBPP treadmill for this type of training.

\section{Elastic tubing}

Elastic tubing may be extremely effective throughout the rehabilitation process. In the initial phase of rehab, it may be used to minimize the amount of weight bearing on the affected limb. An example would be to have the tubing pulling the person onto their unaffected side to avoid excessive loading during gait. Another example would be to use the tubing as an assistive device. This could be done by anchoring the tubing overhead and assisting with hip flexion when the hip flexor must be worked and patterned, but does not have the strength necessary to perform the maneuver in a repetitive fashion. Another example would be to pattern a movement such as a golf swing. In addition to patterning the movement, a deceleration component is required by the trunk and the lead leg as the weight is being transferred from the back foot to the front foot. The third way to use the tubing would be in a resistive fashion. This may be implemented when the goal is to increase strength, power and function, but only after proper patterning has occurred. The last way to use the tubing would be in an overload or towing fashion. The motion may be comparable to the assistive motion, but the speed of the assistance/ towing would be much higher. The speed should never exceed a level that contributes to a total loss of form or compensated patterning. Although this is common practice in speed training, this is also seen when performing multiple angle agility drills. These examples serve as alternatives for clinics which are restricted from purchasing the previously discussed cost prohibitive options.

\section{Return to sport testing and considerations}

\section{Screening programs}

There are many commonly accepted screening programs. Although they may provide some insight and may meet their testing objectives, they may not necessarily provide the answers. The ability to observe motion which is performed in a controlled fashion certainly is valuable. However, full clearance for return to sport must clear movements which are done under sport specific or required workloads, provide a sport specific speed or acceleration/deceleration component, and are measured during different physiologic states of the training period

\section{Clinical clearance}

From a clinical standpoint, it is necessary to identify neuromuscular or articular shortcomings of the patient. A patient presenting with postural imbalances may demonstrate poor
Table 2 Running program in LBPP treadmill (2-3x/week)

Progress to over ground interval running program

\begin{tabular}{llll}
\hline Post Op week & Time (min) & Speed (mph) & Body Weight \\
\hline 13 & Run 1/walk 1 (intervals x5) & 6.0 (or 5 K race pace) & $60 \% \mathrm{BW}$ \\
14 & Run 2/walk 1 (intervals x4) & 6.5 (or 5 K race pace) & $70 \% \mathrm{BW}$ \\
15 & Run 3/walk 1 (intervals x5) & 6.5 (or 5 K race pace) & $80 \% \mathrm{BW}$ \\
16 & Run 4/walk 1 (intervals x 4) & 6.5 (or 5 K race pace) & $85 \% \mathrm{BW}$ \\
\hline
\end{tabular}


articular mechanics during both open and closed chain hip activity. Failed load transfer tests such as the active straight leg raise test [24], the single leg stance test [25] and the squat test [25] will all demonstrate an anterior translation of the femoral head while attempting the movement. The landmark used to identify the anterior translation is the greater trochanter. In addition to performing these three specific failed load transfer tests, a 10 rep triple test is used in our clinic before allowing a patient to initiate a structured land based progressive running program. The three exercises used in the ten rep triple test include the front step down, the single leg squat, and side lying hip abduction Previously, $5 / 5$ for hip abduction manual muscle test (MMT) for the 10 repetitions was required, but observationally it was noted that a person could pass both closed chain tests and marginally fail the open chain part of the test battery. Therefore it is required the patient have at least a 4/5 MMT grade for all hip abduction repetitions. The explanation for this observation could be as simple as open chain endurance weakness or improved neuromuscular control in the closed chain position. All three exercises are incorporated into the phased post-operative protocol, so it certainly would not suggest a learning curve being necessary for the protocol being conducted. Another battery of functional tests utilized for return to sport following hip surgery at the Steadman Clinic [26] includes the single knee bend, lateral agility test, diagonal agility test and forward lunge onto box test. The single knee bend test is performed for up to $3 \mathrm{~min}$, the lateral and diagonal agility tests up to $100 \mathrm{~s}$ and the forward box lunge up to $2 \mathrm{~min}$.

\section{Return to sport testing}

Regardless of the sport, skills most required for return to activity include squatting, running, jumping, landing, cutting, kicking, twisting, throwing and hitting. In many instances, testing measures have evolved based on observational findings of injury mechanism patterns. Testing will assist in determining an athlete's return-to-play status.

Clinical measures such as ROM, joint laxity, and strength measurements are insufficient in determining an athlete's readiness to return to sport [27]. The final phase of rehabilitation requires appropriate transition to performance and higher-sports related activities [28], [29••]. Functional performance tests (FPTs) provide information relative to the functional level of performance necessary in sports competition [30]. Performance and agility testing should demonstrate power, speed, and demands of the sport. This exposes the athlete's readiness perception and actual readiness to return to sport $[28,29 \bullet \bullet]$. Research has determined the hop test to be a reliable and valid tool for determination of safe return-to-play after rehabilitation of a lower extremity (LE) injury. The hop test can be completed in various ways: single leg hop for distance, cross-over hop for distance, triple hop- for distance, six-meter timed hop [27]. A majority of research has been completed in patients status post ACLR to assess limb asymmetries and rule out potential factors for additional LE injury [31]. Limb symmetry index (LSI) provides performance measurements between operated and non-operated lower extremity [32]. LSI $>85 \%$ had been the gold standard for RTP, but more recently Munro and Herrington [27] suggest utilizing $>90 \%$ limb symmetry. The single leg hop test assists in determining deficits in power and postural stability in the LE. Sport specific motions such as cutting require the athlete to generate power and maintain stability on one leg. Proper transfer of kinetic energy is necessary to make short, quick and explosive movements during sport participation. Landing strategies are important to ensure proper load transfer throughout the LE. Deficits in landing may identify weakness in thigh and hip musculature and require attention in neuromuscular training to improve hip and thigh muscle recruitment. Neuromuscular training addresses lateral motion deficits, postural stability, limb force production and control throughout landing $[28,29 \bullet \cdot]$.

Other agility tests that may be utilized are agility $T$ test, figure of 8 test, line drill test and zigzag test. The Agility $T$ test looks at lateral motion deficits, acceleration/deceleration, and directional changes with cutting and running drills $[27,33]$. One trial for each limb is needed for comparison. The push-off limb determines the limb tested. The modified T- test has been shown to have good reliability in National Football League (NFL) combine testing methods [33]. The zigzag run test measures agility, quick change in direction (cutting and turning), and acceleration/deceleration components of activity. This test demonstrates excellent reliability. Line drill tests examine speed, anaerobic activity, and degree of fatigue. Other components of the test are similar to the zigzag test in measuring agility performance, but no literature is reported on its reliability and validity. Figure of 8 test also assesses agility, but lacks standardization of testing in the literature. The degree of difficulty of the test can be altered by changing length and width of cone placement. All mentioned agility tests can assess limb symmetry by comparison on motion and time completion between limbs tested [27]. Functional testing is an important component to return to play criteria. Making function a focus throughout the rehabilitation process helps to ensure goals from basic clinical advancement to more complex movement patterns for sport-specifics are attained and full restoration of normal biomechanics and strength are achieved [34].

\section{Field testing}

It is necessary to perform both a general, as well as a sport specific field test. The general test includes forward running, backward running, side to side shuffle, carioca, cutting, 
accelerating and decelerating at 30, 45, 6090 and $180^{\circ}$. In addition, the person should be able to jump, land, and accelerate without kinetic collapse, as well as run tight figure eights and circles in both directions without apprehension. Since the sport specific test will address the skills necessary for returning to their respective sport, it is encouraged to invite a position coach during the testing, especially if you are not familiar with the details of the skill requirements.

\section{Training considerations}

The squat or a derivative of a squat is another very important position for the athlete to assume for return to sport. Whether you are a basketball player in a defensive position, a fencer in a split squat position, a linebacker in a stance position, or a soccer player about to jump for a header, the most common initial or ready position assumed is a knee bent, head up and back straight position. If the sport skill requires a deep hip flexed position such as an offensive lineman, a rugby player, a sprinter, or a face-off specialist in lacrosse, the demands on the structure of the hip will be even greater and probably compromised, particularly if an overcoverage problem does exist. Therefore, although playing in this position or a derivative of this position may be indicated, training postures may have to be modified. For years, the squat has been the universally accepted exercise for building lower body strength and power. However, recently respected strength and conditioning specialists such as Mike Boyle have begun to use more squat derivative type lifts in their programs in lieu of the squat. Examples may include lunges, split leg squats, sled pulls or pushes and dumbbell snatches. McGill has shifted towards more asymmetric carries, pushes, pulls and lifts [35]. Regardless of the philosophical training change, athletic function at times requires there be two feet firmly on the ground. In many sports, it is from this stable, squat like base that an athlete is asked to be able to control, manipulate or move large uncooperative forces.

It is not recommended that a person perform loaded squat activities for most types of impingement [36•], but it is still important to understand why these are commonly implemented and accepted type lifts. Bentley et al. [37] report that a fast descent coupled with a fast ascent during the squat maneuver maximizes musculoskeletal loading. Kipp et al. [38] showed that torque and rate of torque development at the hip during the pull phase of the clean were highest at greater than $75 \%$ of one repetition maximum (RM). Finally, McBride et al. [39] compared the effects on performance between the squat and box squat. Even with replacing the stretch shortening cycle that occurs with the squat, no significant differences were found in performance. The compelling argument for doing such lifts is for the development of strength and explosive power, which is required for excelling in so many sports. One must consider what lifts are best suited for which hip structure. Some athletes may benefit most with modified power lifts, while others may benefit most from either normal or modified Olympic lifts or derivatives. Certain types of mechanical hip problems may be restricted to some type of symmetrical or asymmetrical functional training.

\section{Conclusion}

The decision to return to sport will usually include input from the athlete, rehabilitation specialist, coach, strength coach, and parent when necessary. The process should be overseen and coordinated by the team physician directing the athlete's care. The decision to return to play should include objective clinical and functional testing, objective clinical exam, subjective sport specific testing and athlete feedback and interaction. It is important that the athlete not only pass the test battery provided, but that it is done without pain, without compensation, and without apprehension. The athlete should always know that until they are able to perform the same exact maneuver in the same exact environment that caused the problem, otherwise they will be left with a cognitive uncertainty as to whether or not they are completely ready to return. This is why it is absolutely necessary to lay out a tentative return to sport program and testing battery at the onset. This will demonstrate to the athlete that a progressive return has been well planned with a tentative date for return in mind. In addition, trust will be established that will ensure open communication and feedback throughout the process.

Disclosure No potential conflicts of interest relevant to this article were reported.

\section{References}

Papers of particular interest, published recently, have been highlighted as:

- Of importance

•- Of major importance

1. Miller M, Ploeg AH, Dibbet TJ, et al. The effects of high volume aquatic plyometric training on vertical jump, muscle power, and torque. J Strength Cond Res. 2010;24:1.

2. Harison RA, Hilman M. Bulstrode: Loading of the lower limb when walking partially immersed: Implications for clinical practice. Physiotherapy. 1992;78:164.

3. - Haupenthal A, Ruschel C, Hubert M et al.: Loading forces in shallow water running in two levels of immersion. J Rehabil Med 2010 July, 42: 664-94. Instruction for water based exercises in 
both waist and chest high water should be done with caution knowing that the expected weight bearing forces can change if the speed of the activity is increased.

4. Butts NK, Tucker M, Greening C. Physiologic responses to maximal treadmill and deep water running in men and women. Am J Sport Med. 1991;19:6122.

5. Kaneda K, Sato D, Wakabayashi H, et al. EMG activity of hip and trunk muscles during deep-water running. J Elecromyogr Kinesiol. 2009;19:1064-70.

6. Cole AJ, Eagleston RE, Moschetti M, et al. Spine pain: Aquatic rehabilitation strategies. J Back and Musculoskel Rehabil. 1994;4:273.

7. Tovin BJ, Wolf SL, Greenfield BN, et al. Comparison of the effects of exercise in water and on land on the rehabilitation of patients with intrarticular anterior cruciate ligament reconstruction. Phys Ther. 1994; 74:22.

8. Cutuk AA, Groppo ER, Quigley EJ, et al. Ambulation in simulated fractional gravity using lower body positive pressure: cardiovascular safety and gait analyses. J Appl Physiol. 2006;101:771-7.

9. Ferber R, Davis IM, Hamill J, et al. Kinetic variables in subjects with previous lower extremity stress fractures. Med Sci Sports Exerc. 2002;34:S5.

10. Gerlach KE, White SC, Burtin HW, et al. Kinetic changes with fatigue and relationship to injury in female runners. Med Sci Sports Exer. 2005;37:657-63.

11. Zifchock RA, Davis I, Hamill J. Kinetic asymmetry in female runners with and without retrospective tibial stress fractures. J Biomech. 2006;39:2792-7.

12. Eastlack RK, Hargens AR, Groppo ER, et al. Lower body positivepressure exercise after knee surgery. Clin Orth Relat Res. 2005;431:213-9.

13. Wilk KE, Macrina LC, Reinold MM. Rehabilitation following microfracture of the knee. Cartilage. 2010;1:96-107.

14. Grabowski A, Kram R. Effects of velocity and weight support on ground reaction forces and metabolic power during running. $\mathrm{J}$ Appl Biomech. 2008;24:288-97.

15. Grabowski A. Metabolic and biomechanical effects of velocity and weight support using a lower body positive pressure device during walking. Arch Phys Med Rehab. 2010;91:951-7.

16. Hoffman MD, Donaghe HE. Exercise responses during partial body-weight supported treadmill walking and running in healthy individuals. Arch Phys Med Rehab. 2011;92:960-6.

17. Liebenberg J, Scharf J, Forrest D, et al. Determination of muscle activity during running at reduced body weight. J Sports Sciences. 2011;29:207-14.

18. Liebenberg J, Scharf J, Forrest D, Dufek J, Mercer JA: Muscle activity during running at reduced body weight (University of Nevada, Las Vegas), presented at southwest meeting of ACSM, San Diego, October 2009.

19. Saxena A, Granot A: Use of an anti-gravity treadmill in the rehabilitation of the operated Achilles tendon: A pilot study. Presented at American College of Foot and Ankle Surgeons Annual Conference, Las Vegas, NV, February 2010.

20. Saxena A, Granot A: Use of an anti-gravity treadmill in the rehabilitation of the operated achilles tendon: a pilot study. Accepted for publication to J Foot and Ankle Surgery, In Press

21. Daniels J. Daniels' running formula. Champaign: Human Kinetics; 2005.

22. Weyand PG, Sternlight DB, Bellizzi MJ, et al. Faster top running speeds are achieved with greater ground forces not more rapid leg movements. J Appl Physiol. 2000;89:1991-9.
23. Roberts TJ, Kram R, Weyand PG, et al. Energetics of bipedal running I. Metabolic cost of generating force. J Exp Biol. 1998;201:2745-51.

24. Mens JM, Vleeming, Snijders CJ, et al. The active straight leg raising test and mobility of the pelvic joints. Eur Spine J. 1999;8:468-73.

25. Lee D, Lee LJ. Techniques and tools for assessing the lumbopelvic-hip complex. In: Lee D, editor. The Pelvic Girdle. New York: Elsevier; 2011. p. 173-282.

26. Wahoff M, Ryan M. Rehabilitation after hip femoroacetabular impingement arthroscopy. Clin Sports Med. 2011;30:463-82.

27. Munro AG, Herrington LC. Between session reliability of four hop tests and the agility T-test. J Strength Cond Res. 2011;25:1470-7.

28. Myer GD, Paterno MV, Ford KR, et al. Rehabilitation after anterior cruciate ligament reconstruction: criteria-based progression through the return-to-sport phase. J Orthop Sports Phys Ther. 2006;36:385-402.

29. •• Myer GD, Schmitt LC, Brent JL, et al.: Utilization of modified NFL combine testing to identify functional deficits in athletes following ACL reconstruction. J Orthop Sports Phys Ther. 2011, 41: 377-388. On-field and performance testing can be an important tool in identifying lower limb performance deficits throughout the lower extremity chain. Single limb tasks demonstrate a higher incidence of deficit than bipedal testing.

30. Lephart S, Perrin D, Fu F, et al. Relationship between selected physical characteristics and functional capacity in the anterior cruciate ligament insufficient athlete. J Orthop Sports Phys Ther. 1992;16:174-81.

31. Kvist J. Rehabilitation following anterior cruciate ligament injury: current recommendations for sports participation. Sports Med. 2004;34:269-80

32. Barber SD, Noyes FR, Mangine RE, et al. Quantitative assessment of functional limitations in normal and anterior cruciate ligamentdeficient knees. Clin Orthop Relat Res. 1990;255:204-14.

33. Hickey KC, Quaman CE, Myer GD, et al. Methodological report: dynamic field tests used in an NFL combine setting to identify lower-extremity functional asymmetries. J Strength Cond Res. 2009;23:2500-6.

34. Austin, GP: Functional Testing and Return to Activity. in Scientific Foundations and Principles of Practice in Musculoskeletal Rehabilitation. Saunders Elsevier 2007; 633-664.

35. McGill S: Training maximum performance: what we learn from the fastest and strongest athletes. Presentation at National Strength and Conditioning Association Annual conference, July 2011: Las Vegas.

36. - Lamontagne M, Brisson N, Kennedy MJ, et al. Preoperative and postoperative lower extremity joint and pelvic kinematics during maximal squatting of patients with cam femoroacetabular impingement. J Bone Joint Surg Am 201193 Suppl 2: 40-5. Deep squatting activities should be avoided in patients diagnosed with cam femoroacetabular impingement. This is especially true for the person who has had the osseous deformity corrected, but presents with a similar structured hip on an asymptomatic side.

37. Bentley JR, Amonette WE, DeWitt JK, et al. Effects of different lifting cadences on ground reaction forces during the squat exercise. J Strength Cond Res. 2010;24:1414-20.

38. Kipp K, Harris C, Sabick M. Lower extremity biomechanics during weightlifting exercise vary across joint and load. J Strength Cond Res. 2011;25:1229-34.

39. McBride JM, Skinner JW, Schafer PC, et al. Comparison of kinetic variables and muscle activity during a squat vs a box squat. J Strength Cond Res. 2010;24:3195-9. 